\title{
EFEITOS DOS EXERCÍCIOS AQUÁTICOS NA OSTEOARTRITE DO QUADRIL OU JOELHO: REVISÃO
}

\author{
Maria Rita Masselli ${ }^{1}$, Ângela Kazue Morita $^{2}$, Célia Aparecida Stellutti Pachioni ${ }^{1}$, Dalva Minonroze \\ Albuquerque Ferreira ${ }^{1}$
}

${ }^{1}$ Docente do Departamento de Fisioterapia, ${ }^{2}$ Discente do Curso de Especialização em Fisioterapia da FCT/UNESP, Presidente Prudente, SP.

\section{RESUMO}

A osteoartrite (OA) de joelho ou quadril é uma doença articular degenerativa comum que afeta o indivíduo em múltiplas dimensões: do nível orgânico até o social. A doença está associada com dor e rigidez articular, deformidade e progressiva perda da função. Esta revisão teve por objetivo reunir informações a respeito dos benefícios proporcionados pela atividade física realizada no meio aquático, especialmente nos casos em que a ação da gravidade gera dificuldade e dor para a realização dos exercícios. Foram realizadas pesquisas nas seguintes bases de dados eletrônicas com acesso online: Ibecs, Lilacs, Medline, Pubmed e Scielo e incluídos ensaios clínicos randomizados, utilizando o cruzamento entre os descritores: osteoartrite do joelho, osteoartrite do quadril, hidroterapia, reabilitação e terapia por exercício, nos idiomas inglês, espanhol e português, referentes ao período de 2001 a 2011. Foram selecionados dez estudos, sendo que em seis comparou-se os efeitos dos exercícios realizados no solo com os realizados na água em portadores de OA. O grupo de exercícios aquáticos apresentou-se superior quanto à redução da dor, à adesão ao tratamento e à redução da proporção de gordura corporal. O grupo de exercícios realizados no solo apresentou melhores resultados para os ganhos da força muscular e nível de satisfação. A hidroterapia proporcionou diversos benefícios para indivíduos com OA de quadril ou joelho e a redução da dor foi o seu principal efeito terapêutico, além de ser uma atividade bem aceita, beneficiando os indivíduos com diferentes graus de OA.

Palavras-chave: osteoartrite do joelho, osteoartrite do quadril, hidroterapia, reabilitação, terapia por exercício.

\section{EFFECTS OF AQUATIC EXERCISE ON HIP OR KNEE OSTEOARTHRITIS: A REVIEW}

\begin{abstract}
Osteoarthritis (OA) of the knee or hip is a common degenerative joint disease that affects the individual in multiple dimensions: from organic level to the social. The disease is associated with pain and joint stiffness, deformity, and progressive loss of function. This review aimed to gather information about the benefits provided by the physical activity performed in the aquatic environment, especially in cases where the action of gravity causes pain and difficulty for the exercises. Surveys were conducted in the following electronic databases accessible online: Ibecs, Lilacs, Medline, Pubmed and Scielo and included randomized controlled trials using the intersection between the descriptors: knee osteoarthritis, hip osteoarthritis, hydrotherapy, rehabilitation and exercise therapy, in English, Spanish and Portuguese, for the period 2001 to 2011. Ten studies were selected being six compared the effects of exercise performed in the soil with the water conducted in patients with OA. The aquatic exercise group showed himself superior in reducing pain, treatment adherence and reduce the proportion of body fat. The exercise group performed in soil showed better results for the gains in muscle strength and level of satisfaction. Hydrotherapy has provided many benefits for individuals with $O A$ of the hip or knee and pain reduction was the main therapeutic effect, besides being a well-accepted activity, benefiting individuals with varying degrees of $O A$.

Keywords: knee osteoarthritis, hip osteoarthritis, hydrotherapy, rehabilitation, exercise therapy.
\end{abstract}




\section{INTRODUÇÃO}

A Osteoartrite (OA) é uma doença articular crônica localizada e caracteriza-se pela degeneração da cartilagem articular, hipertrofia óssea marginal, redução do espaço articular e alterações da membrana sinovial (BENNELL; HINMAN, 2011; ESCALANTE et al., 2010; ESCALANTE et al., 2011). Afeta grande parcela da população, especialmente os idosos (BIELEMAN et al., 2010; GELBER, 2010; LEE; SHMERLING, 2008; VAN DIJK et al., 2008) e indivíduos com sobrepeso e obesidade (BIELEMAN et al., 2010; GELBER, 2010; VAN DIJK, 2008). Outros fatores de risco podem ser citados, como o gênero feminino, comprometimento articular prévio e fraqueza muscular dos membros inferiores (BENNELL; HINMAN, 2011). Trata-se de uma das doenças com as maiores taxas de comorbidades, frequentemente associada à diabetes, hipertensão, desordens cardiovasculares e sobrepeso (VAN DIJK et al., 2008; REEUWIJK et al., 2010).

A dor é o principal sintoma e não há uma correlação clara entre a sua intensidade e a gravidade das alterações observadas a partir do diagnóstico por imagem. A dor associada à rigidez articular, instabilidade, edema e fraqueza muscular dificulta a realização das atividades de vida diária (AVDs) (BENNELL; HINMAN, 2011), caracterizando a perda de função (BENNELL; HINMAN, 2011; ESCALANTE et al., 2010; LEE; SHMERLING, 2008; VAN DIJK et al., 2008; KERSTEN et al., 2010), o que prejudica a qualidade de vida (BENNELL; HINMAN, 2011; ESCALANTE et al., 2010; PISTERS et al., 2010) e aumenta os riscos de mortalidade e morbidade (ESCALANTE et al., 2010). A mobilidade encontra-se limitada em $80 \%$ dos pacientes e 25\% são incapazes de realizar as AVDs (BENNELL; HINMAN, 2011; ESCALANTE et al., 2010).
As articulações mais afetadas são quadril e joelho, seguidas pelas da mão e da coluna (BENNELL; HINMAN, 2011; ESCALANTE et al., 2010). Ao comprometer os membros inferiores, as limitações da função física podem prejudicar o desenvolvimento das atividades laborais (BIELEMAN et al., 2010). Ocorre um aumento do número de artroplastias de quadril e joelho a cada ano em todo o mundo, havendo uma estimativa de duplicação do número de casos até 2016 (GILL et al., 2009).

Atualmente existem basicamente três formas de tratamento da OA: cirúrgico (ESCALANTE et al., 2010; PISTERS et al., 2010), conservador utilizando-se ou não de fármacos (ESCALANTE et al., 2010; LEE; SHMERLING, 2008; PISTERS et al., 2010).

Os efeitos do tratamento não farmacológico para a OA são semelhantes aos do tratamento farmacológico, o que sugere a importância de sua recomendação. As medidas não farmacológicas incluem as órteses (RANNOU; POIRAUDEAU, 2010), exercícios físicos (ESCALANTE et al., 2010; RANNOU; POIRAUDEAU, 2010; AGEBERG et al., 2010), dieta (LEE; SHMERLING, 2008; RANNOU; POIRAUDEAU, 2010; GRAINGER; CICUTTINI, 2004), acupuntura (LEE; SHMERLING, 2008; KERSTEN et al., 2010), imersão em águas termais (LEE; SHMERLING, 2008; YURTKURAN et al., 2006) e a educação dos pacientes (RANNOU; POIRAUDEAU, 2010; GRAINGER; CICUTTINI, 2004). Nos casos avançados as artroplastias parciais ou totais de quadril e joelho tornam-se praticamente inevitáveis (PISTERS et al., 2010).

Os exercícios físicos são amplamente prescritos para a $O A$ de membros inferiores (ESCALANTE et al., 2010; RANNOU; POIRAUDEAU, 2010; AGEBERG et al., 2010; FRENCH et al., 2009), podendo melhorar a amplitude de movimento, força muscular, 
flexibilidade, desempenho aeróbico e propriocepção (RANNOU; POIRAUDEAU, 2010; GRAINGER; CICUTTINI, 2004; BLACKHAM et al., 2008), além de reduzir a dor e melhorar a função nas AVDs (ESCALANTE et al., 2010; REEUWIJK et al., 2010; BLACKHAM et al., 2008; BOSOMWORTH, 2009). Os exercícios aquáticos têm sido indicados (BENNELL; HINMAN, 2011; ESCALANTE et al., 2010; SILVA et al., 2008), especialmente nos casos em que a ação da gravidade gera dificuldade e dor para a realização dos exercícios. O meio aquático permite mobilização ativa precoce e o fortalecimento dinâmico (SILVA et al., 2008), sendo a hidroterapia frequentemente prescrita nos casos de osteoartrite dos membros inferiores por serem articulações de sobrecarga (LEE; SHMERLING, 2008).

Considerando a importância desta forma de intervenção o presente estudo teve por objetivo reunir informações a respeito dos benefícios proporcionados pela atividade física realizada no meio aquático nos indivíduos com OA de quadril ou joelhos, no sentido de contribuir para melhor entendimento de pesquisadores e clínicos que trabalham com esta população.

\section{MÉTODOS}

A revisão da literatura foi realizada em bases de dados eletrônicas com acesso online: Ibecs, Lilacs, Medline, Pubmed e Scielo. Foram incluídos ensaios clínicos randomizados, utilizando o cruzamento entre os descritores: osteoartrite do joelho, osteoartrite do quadril, hidroterapia, reabilitação e terapia por exercício, nos idiomas inglês, espanhol e português, referentes ao período de 2001 a 2011. Foram selecionados 10 artigos (Tabela 1), nos quais quatro avaliaram a população com OA de joelho (SILVA et al., 2008; LIM et al., 2010; LUND et al., 2008; WYATT et al., 2001) e seis com OA de quadril e/ou joelho (GILL et al., 2009; COCHRANE et al., 2005; FOLEY et al., 2003; FRANSEN et al., 2007; HINMAN et al., 2007; WANG et al., 2007).

\section{RESULTADOS}

A maioria dos estudos comparou os efeitos dos exercícios realizados no solo com os realizados na água em portadores de OA de quadril e/ou joelho (GILL et al., 2009; SILVA et al., 2008; LIM et al., 2010; LUND et al., 2008; WYATT et al., 2001; FOLEY et al., 2003). O grupo de exercícios aquáticos (GEA) apresentou-se superior quanto à redução da dor (GILL et al., 2009; SILVA et al., 2008; LIM et al., 2010; WYATT et al., 2001), à adesão ao tratamento (SILVA et al., 2008) e à redução da proporção de gordura corporal (LIM et al., 2010). O grupo de exercícios realizados no solo (GES) apresentou melhores resultados para os ganhos da força muscular (LUND et al., 2008; FOLEY et al., 2003) e nível de satisfação (FOLEY et al., 2003). 
Tabela 1. Caracterização dos estudos encontrados na literatura.

\begin{tabular}{|c|c|c|c|c|c|}
\hline Autores/ano & & \multicolumn{2}{|c|}{ População } & Protocolo & Conclusão \\
\hline Wyatt et al., 2001 & \multicolumn{3}{|c|}{$\begin{array}{l}\text { GES+GEA } \\
\mathrm{N}=46\end{array}$} & $\begin{array}{l}45^{\prime}-60^{\prime} / 3 \text { vezes } / 6 \\
\text { semanas }\end{array}$ & $\begin{array}{l}\text { Dor inferior para o GEA e } \\
\text { não houve diferenças nos } \\
\text { demais aspectos avaliados. }\end{array}$ \\
\hline Foley et al., 2003 & $\begin{array}{l}\text { GEA } \\
N=35 \\
O A Q / J\end{array}$ & $\begin{array}{l}\text { GES } \\
N=32 \\
O A Q / J\end{array}$ & $\begin{array}{c}\mathrm{GC} \\
\mathrm{N}=35 \\
\mathrm{OAQ} / \mathrm{J}\end{array}$ & $\begin{array}{l}30 ' / 3 \text { vezes } / 6 \\
\text { semanas }\end{array}$ & $\begin{array}{l}\text { GES superior no ganho de } \\
\text { força muscular e GEA } \\
\text { melhor condicionamento. }\end{array}$ \\
\hline Cochrane et al., 2005. & $\begin{array}{l}\text { GEA } \\
N=153 \\
O A Q / J\end{array}$ & & $\begin{array}{c}G C \\
N=159 \\
O A Q / J\end{array}$ & $\begin{array}{c}60 ' / 2 \text { a } 3 \text { vezes } / 12 \\
\text { meses }\end{array}$ & $\begin{array}{l}\text { Redução significante da dor } \\
\text { e melhora da função física. }\end{array}$ \\
\hline Fransen et al., 2007. & $\begin{array}{l}\text { GEA } \\
N=55 \\
O A Q / J\end{array}$ & $\begin{array}{l}\text { Tai Chi } \\
\text { Chuam } \\
N=56 \\
\text { OAQ/J }\end{array}$ & $\begin{array}{l}G C \\
N=51 \\
O A Q / J\end{array}$ & $\begin{array}{l}60 ' / 2 \text { vezes } / 12 \\
\text { semanas }\end{array}$ & $\begin{array}{l}\text { Hidroterapia superior } \\
\text { quanto ao alívio da dor, } \\
\text { melhora da capacidade } \\
\text { física e nível de adesão. }\end{array}$ \\
\hline Wang et al., 2007. & $\begin{array}{l}\text { GEA } \\
N=20 \\
O A Q / J\end{array}$ & & $\begin{array}{c}\mathrm{GC} \\
\mathrm{N}=18 \\
\mathrm{OAQ} / \mathrm{J}\end{array}$ & $\begin{array}{l}50 ' / 3 \text { vezes } / 12 \\
\text { semanas }\end{array}$ & $\begin{array}{c}\text { Melhora da flexibilidade e } \\
\text { força dos membros } \\
\text { inferiores e da capacidade } \\
\text { aeróbica. }\end{array}$ \\
\hline Hinman et al., 2007. & $\begin{array}{l}\text { GEA } \\
\mathrm{N}=36 \\
\mathrm{OAQ} / \mathrm{J}\end{array}$ & & $\begin{array}{c}G C \\
N=35 \\
O A Q / J\end{array}$ & $\begin{array}{l}45^{\prime}-60^{\prime} / 2 \text { vezes } / 6 \\
\text { meses }\end{array}$ & $\begin{array}{l}\text { Melhora discreta da dor, } \\
\text { rigidez, aptidão física, força } \\
\text { muscular e qualidade de } \\
\text { vida. }\end{array}$ \\
\hline Silva et al., 2008. & $\begin{array}{c}\text { GEA } \\
\mathrm{N}=32 \\
\mathrm{OAJ}\end{array}$ & & $\begin{array}{l}\text { GES } \\
\mathrm{N}=32 \\
\mathrm{OAJ}\end{array}$ & $\begin{array}{l}50 ' / 3 \text { vezes } / 18 \\
\text { semanas }\end{array}$ & $\begin{array}{l}\text { Hidroterapia superior no } \\
\text { alívio da dor antes e após } \\
50 \text { FWT e redução no } \\
\text { consumo de drogas e } \\
\text { melhor adesão. }\end{array}$ \\
\hline Lund et al., 2008. & $\begin{array}{c}\text { GEA } \\
\mathrm{N}=27 \\
\mathrm{OAJ}\end{array}$ & $\begin{array}{c}\text { GES } \\
\mathrm{N}=25 \\
\mathrm{OAJ}\end{array}$ & $\begin{array}{l}\mathrm{GC} \\
\mathrm{N}=27 \\
\mathrm{OAJ}\end{array}$ & $\begin{array}{l}50 ' / 2 \text { vezes } / 8 \\
\text { semanas }\end{array}$ & $\begin{array}{c}\text { Redução da dor e aumento } \\
\text { da força muscular no GES. } \\
\text { Sem benefícios clínicos no } \\
\text { GEA. }\end{array}$ \\
\hline Gill et al., 2009. & $\begin{array}{l}\text { GEA } \\
N=42 \\
O A Q / J\end{array}$ & & $\begin{array}{l}\text { GES } \\
N=40 \\
O A Q / J\end{array}$ & $\begin{array}{l}60 ' / 2 \text { vezes } / 6 \\
\text { semanas }\end{array}$ & $\begin{array}{l}\text { Melhoraram a função } \\
\text { anterior à artroplastia de } \\
\text { quadril ou joelho. }\end{array}$ \\
\hline Lim et al., 2010. & $\begin{array}{c}\text { GEA } \\
N=26 \\
O A J\end{array}$ & $\begin{array}{l}\text { GES } \\
N=25 \\
\text { OAJ }\end{array}$ & $\begin{array}{c}\mathrm{GC} \\
\mathrm{N}=24 \\
\mathrm{OAJ}\end{array}$ & 3 vezes/8 semanas & $\begin{array}{l}\text { Hidroterapia reduziu a dor e } \\
\text { é alternativa eficaz para } \\
\text { obesos com OAJ com } \\
\text { dificuldades para se } \\
\text { exercitar. }\end{array}$ \\
\hline
\end{tabular}

Legendas: GES: grupo de exercícios no solo; GEA: grupo de exercícios aquáticos; OAJ: Osteoartrite do Joelho; OAQ/J= Osteoartrite do quadril e/ou joelho; GC: grupo controle. 
Três estudos Cochrane, Davey e Edwards (2005), Hinman et al. (2007) e Wang et al. (2007) compararam os benefícios da hidroterapia com os do grupo controle (GC). Entre eles, foi avaliada a eficácia de um programa comunitário de hidroterapia para idosos (COCHRANE et al., 2005). O GEA apresentou significante redução da dor e melhora da função física, além de apontar favorável custo-benefício, utilizando o escore de dor da Western Ontario and McMaster Universities OA index (WOMAC) como parâmetro. Wang et al. (2007) observaram ganhos em curto prazo quanto à flexibilidade e força muscular dos membros inferiores e melhora da capacidade aeróbica. Por outro lado, não houve melhora significativa da dor e da função física. Hinman et al. (2007) verificaram elevada adesão ao tratamento e ganhos discretos quanto à redução da dor, função física, qualidade de vida e força muscular.

Os efeitos da hidroterapia foram comparados aos do Tai Chi Chuam em portadores de OA de quadril e/ou joelho no estudo de (FRANSEN et al., 2007). A hidroterapia foi mais efetiva na redução da dor, além de proporcionar evidentes ganhos da performance física e ser mais aceitável pelos pacientes em comparação ao Tai Chi Chuam.

A seguir serão apontados os principais benefícios apontados pelos estudos e os argumentos utilizados pelos autores.

\section{Intensidade da dor}

Este benefício foi atribuído à flutuabilidade proporcionada pelo meio aquático, que ao reduzir a descarga de peso sobre a articulação, diminui a percepção da intensidade da dor e a melhora o movimento funcional e da capacidade de se exercitar (WYATT et al., 2001; HINMAN et al., 2007). A temperatura e a pressão hidrostática também são responsáveis por este efeito, sendo que o calor promove o relaxamento muscular e reduz a tensão ao redor articulação, otimizando o movimento. Entretanto, a imersão em água aquecida deve ser complementada pela realização de exercícios para o fortalecimento dos músculos dos membros inferiores, priorizando o quadríceps femoral, para que o alívio da dor seja efetivo (SILVA et al., 2008; HINMAN et al., 2007).

A redução do uso de medicamentos analgésicos foi um parâmetro da eficácia do tratamento aquático, além de representar uma vantagem por diminuir os riscos de complicações gastrointestinais e renais em decorrência do seu consumo (SILVA et al., 2008). Neste mesmo estudo, a hidroterapia foi superior ao GES para aliviar a dor antes e após o 50 FWT (Foot Walk Test), que representa um percurso funcional presente nas AVDs. Isto demonstra a influência positiva na realização das atividades cotidianas.

Até mesmo nos casos graves de OA de quadril e/ou joelho, em que a cirurgia eletiva de substituição era necessária, a hidroterapia, assim como os exercícios no solo, foram efetivos para reduzir a dor e melhorar a função. A hidroterapia foi superior com relação à redução da dor à curto prazo, avaliada imediatamente após as sessões e no dia seguinte a sua realização (GILL et al., 2009).

No estudo em que não houve modificação da dor, um dos motivos relacionados foi o uso da Escala Visual Analógica (EVA) como instrumento de avaliação da dor geral do corpo e não dos movimentos ou atividades específicos da articulação acometida. Logo, sugeriu-se que maior atenção fosse dada quanto à forma de avaliação da dor (WANG et al., 2007). Contudo, outros estudos também utilizaram a EVA (SILVA et al., 2008; LUND et al., 2008; WYATT et al., 2001; HINMAN et al., 2007), assim como a WOMAC (GILL et al., 2009; SILVA et al., 2008; COCHRANE et al., 2005; FOLEY et al., 2003; FRANSEN et al., 2007; HINMAN et al., 2007), e ambas detectaram melhoras no nível da dor. 


\section{Função física}

Os ganhos na função física foram observados por meio de testes funcionais. O GEA obteve melhor desempenho no Teste de Caminhada de 6 minutos (TC6) para a distância percorrida e o GES para a velocidade quando comparados ao GC (FOLEY et al., 2003). Outros estudos verificaram incrementos de 10,9\% da distância percorrida no TC6 (WANG et al., 2007) e de $9 \%$ nos minutos do 1-mile walk test (WYATT et al., 2001) pelos GEAs.

Verificou-se ainda que a severidade da OA poderia influenciar os ganhos da função física do GEA. Neste estudo foram encontrados apenas ganhos discretos da função física e a gravidade da OA foi apontada como causa. Logo, sugeriu-se que a hidroterapia seria mais eficaz nos casos menos severos de OA. Os pequenos ganhos obtidos foram atribuídos aos exercícios de estabilização central com o uso de posicionamentos funcionais (HINMAN et al., 2007).

Em contraste, um estudo composto por sujeitos com OA grave, apresentou ganho funcional físico para o GEA. Os autores compararam a hidroterapia com o Tai Chi Chuam e obtiveram melhoras na função física em ambos os grupos, comparados com o controle. O GEA apresentou melhoras significativas da depressão e estresse, componente físico do SF-12 e das 3 mensurações da performance física, enquanto o grupo Tai Chi Chuam apresentou melhora apenas no stair climb test. Comparados ao controle, somente o GEA apresentou melhora significativa do componente físico do SF-12 e da performance física. O meio aquático foi apontado como uma possibilidade de sentir menos dor para realizar atividades físicas, o que justificaria os ganhos funcionais (FRANSEN et al., 2007).

\section{Força muscular}

Um programa de exercícios resistidos apontou que os exercícios no solo foram mais efetivos no ganho de força muscular, enquanto no meio aquático o condicionamento aeróbico foi melhor estimulado. A hidroterapia proporcionou um ambiente em que os participantes puderam se exercitar aerobicamente, em maiores intensidades do que no solo, em decorrência da redução da descarga de peso. Porém, os exercícios no solo são recomendados para melhorar a estabilidade e a capacidade de absorção de choque por meio da força muscular, pois permitem descarga de peso durante as contrações musculares excêntricas e a progressão da resistência é melhor aplicada, o que já não é possível no meio aquático (HALBERT et al., 2003).

A hidroterapia poderia aumentar a capacidade aeróbica nos pacientes com OA, que tipicamente possuem baixo condicionamento cardiovascular, explicando o significante aumento da função física mesmo sem os significantes ganhos de força. Ainda, os participantes deste estudo apresentavam graus severos de OA e o programa intenso de exercícios aquáticos, quanto ao número de repetições e a velocidade de execução, mostrou-se benéfico até mesmo nos casos em que a artroplastia se fazia necessária (HALBERT et al., 2003).

O estudo de Lund et al. (2008) também observou melhora significativa da força muscular global para o GES enquanto houve um detrimento no GEA, ambos quando comparados ao GC, registrada após 3 meses do término do programa. Este achado foi justificado pela possibilidade do GES ter continuado algum tipo de exercício de fortalecimento em casa. Já o GEA não apresentou aumento de força provavelmente em decorrência da pequena resistência oferecida pelos exercícios e o programa não deve ter estimulado a sua realização em casa. 
A fraqueza dos extensores do joelho, especialmente 0 quadríceps femoral está associado com o desenvolvimento e progressão da OA de joelho. Assim, os ganhos da flexibilidade e da força muscular do quadril e joelho observados no grupo hidroterapia foi um resultado promissor para prevenir as disfunções associadas à OA no decorrer da vida (WANG et al., 2007).

Além do quadríceps femoral, o fortalecimento dos músculos abdutores do quadril foi considerado fundamental para a redução da força compressiva e, consequentemente, da redução da dor. Neste estudo encontrou-se um discreto aumento de força dos músculos abdutores do quadril e não houve ganhos consideráveis para o quadríceps. A falta de equipamentos que oferecessem resistência poderia ter conduzido estes resultados. Contudo, o seu emprego elevaria os custos, podendo reduzir a adesão ao tratamento. Logo, sugeriram que a resistência fosse aplicada por meio das propriedades hidrostáticas e hidrodinâmicas da água, ou seja, reduzindo a profundidade de imersão ou elevando a turbulência por aumento da velocidade ou área de contato (HINMAN et al., 2007).

\section{Adesão ao tratamento}

Nos estudos que avaliaram a adesão ao tratamento predominante, a hidroterapia apresentou altos índices de participação dos sujeitos nos programas. O estudo de Hinman et al. (2007) apresentou a maior taxa de adesão $(99,3 \%)$ e atribuiu o fato à monitoria prestada ao grupo associado à participação na pesquisa científica. Oitenta e quatro por cento dos participantes continuaram alguma atividade física aquática independentemente após 6 meses do término do programa.

Quando comparado ao solo (81\%), a hidroterapia (96\%) também apresentou maior adesão. E, se não fosse a dificuldade de acesso a esta forma de tratamento no sistema público de saúde poderia ter havido ainda maior participação (SILVA et al., 2008).

Com relação ao Tai Chi Chuam (61\%), a hidroterapia (81\%) também foi superior, justificado pelo fato dos exercícios do Tai Chi Chuam serem muito difíceis e dolorosos; em contrapartida, o meio aquático proporcionou redução da dor para a sua realização (FRANSEN et al., 2007).

Apenas o estudo de Cochrane et al. (2005) apresentou baixa taxa de adesão (53,5\%) durante o programa, sendo que apenas $18 \%$ mantiveram as atividades 6 meses após o seu término. A principal razão esteve associada ao fato da população avaliada ser composta por idosos e por este motivo, a presença de patologias associadas, a inaptidão e a dependência de um membro da família foram as principais causas da baixa taxa de participação. Aqueles que não aderiram ao programa apresentaram maior nível de dor, menor função social e nível de vitalidade e uma pior tendência à saúde comparada há um atrás quando aderiram ao tratamento. Assim, os autores sugeriram que para uma proporção significante de portadores de OA a realização regular de atividades físicas não era uma opção viável de tratamento.

\section{Peso corporal}

Apenas o estudo de Lim et al. (2010) analisou os efeitos dos programas de exercícios, realizados no solo ou na água, sobre a gordura corporal em obesos com OA de joelhos. O GEA apresentou redução significante da proporção de gordura corporal, levando a uma discreta redução do IMC, porém não significante. Ainda, para este mesmo grupo, observou-se melhora da dor, inaptidão e qualidade de vida. A diminuição no nível de dor foi significantemente maior no GEA comparado ao GES. Assim, o meio aquático, ao 
proporcionar a redução da dor, possibilita aos pacientes obesos realizarem exercícios ativos.

\section{CONCLUSÃO}

A hidroterapia traz diversos benefícios para portadores de OA, sendo que os estudos avaliados demonstraram que a redução da dor foi o seu principal efeito terapêutico, além de ser uma atividade bem aceita, com elevados níveis de adesão. Ainda, pode contribuir com a redução de gordura corporal, melhorar a função física e a capacidade aeróbica, beneficiando os indivíduos com diferentes graus de OA. Desta forma, a hidroterapia poderia ser uma forma de tratamento para indivíduos portadores de OA de quadril ou joelho, especialmente os obesos, com limitação das atividades funcionais em decorrência da dor.

\section{CONFLITO DE INTERESSE}

Os autores declaram não haver qualquer potencial conflito de interesse que possa intervir na imparcialidade deste trabalho científico.

\section{REFERÊNCIAS}

Ageberg E, Link A, Roos EM. Feasibility of neuromuscular training in patients with severe hip or knee OA: the individualized goal-based NEMEX-TJR training program. BMC Musculoskelet Disord. 2010;11: $126 . \quad$ DOI http://dx.doi.org/10.1186/1471-2474-11-126

Bennell KL, Hinman RS. A review of the clinical evidence for exercise in osteoarthritis of the hip and knee. J Sci Med Sport. 2011;14: 4-9. DOI http://dx.doi.org/10.1016/i.jsams.2010.08.002

Bieleman HJ, Van Ittersum MW, Groothoff JW, Oostveen JCM; Oosterveld FGJ; van der Schans CP; Soer R; Reneman MF. Functional capacity of people with early osteoarthritis: a comparison between subjects from the cohort hip and cohort knee (CHECK) and healthy ageing workers. Int Arch Occup Environ Health. 2010;83: 913-21. DOI http://dx.doi.org/10.1007/S00420-010-0541-3

Blackham J, Garry JP, Cummings DM, Russell RG, Dealleaume L. Does regular exercise reduce the pain and stiffness of osteoarthritis? J Fam Pract. 2008;57(7): 476-7.
Bosomworth NJ. Exercise and knee osteoarthritis: benefit or hazard? Can Fam Physician. 2009;55(9): 871-8.

Cochrane T, Davey RC, Edwards SMM. Randomised controlled trial of the costeffectiveness of water-based therapy for lower limb osteoarthritis. Health Technol Assess. 2005; 9(31): 266-74.

Escalante Y, Saavedra JM, García-Hermoso A, Silva AJ, Barbosa TM. Physical exercise and reduction of pain in adults with lower limb osteoarthritis: A systematic review. J Back Musculoskelet Rehabil. 2010;23(4): 175-86. DOI http://dx.doi.org/10.3233/BMR-2010-0267

Escalante Y, García-Hermoso A, Saavedra JM. Effects of exercise on functional aerobic capacity in lower limb osteoarthritis: a systematic review. J Sci Med Sport. 2011;14(3): 190-8. DOI http://dx.doi.org/10.1016/j.jsams.2010.10.004

French HP, Cusack T, Brennan A, White B, Gilsenan C, Fitzpatrick M, O'Connell P, David K, FitzGerald O, McCarthy GM. Exercise and manual physiotherapy arthritis research trial (EMPART): a multicentre randomised controlled trial. BMC Musculoskelet Disord. 2009;10: 9. DOI http://dx.doi.org/10.1186/1471-2474-10-9

Foley A, Halbert J, Hewitt T, Crotty M. Does hydrotherapy improve strength and physical function in patients with osteoarthritis - a randomized controlled trial comparing a gym based and a hydrotherapy based strengthening programme. Ann Rheum Dis. 2003;62(12): 11627. DOI http://dx.doi.org/10.1136/ard.2002.005272

Fransen M, Nairn L, Winstanley J, Lam P, Edmonds J. Physical activity for osteoarthritis management: a randomized controlled clinical trial evaluating hydrotherapy or Tai Chi classes. Arthritis Rheum. 2007;57(3): 407-14.

Gelber AC. Breaking into a Sweat... and Risk of Osteoarthritis! J Rheumatol. 2010;38(2): 188-90. DOI http://dx.doi.org/10.3899//rheum.101326

Gill SD, McBurney $H$, Schulz DL. Land-based versus pool-based exercise for people awaiting joint replacement surgery of the hip or knee: results of a randomized controlled trial. Arch Phys Med Reabil. 2009;90: 388-94. DOI http://dx.doi.org/10.1016/j.apmr.2008.09.561

Grainger R, Cicuttini FM. Medical management of osteoarthritis of the knee and hip joints. MJA. 2004;180: 232-6.

Hinman RS, Heywood SE, Day A. Aquatic physical therapy for hip and knee osteoarthritis: 
results of a single-blind randomized controlled trial. Phys Ther. 2007;87: 32-43.

Kersten P, White PJ, Tennant A. The Visual Analogue WOMAC 3.0 scale-internal validity and responsiveness of the VAS version. BMC Musculoskelet Disord. 2010;11: 80. DOI http://dx.doi.org/10.1186/1471-2474-11-80

Lee YC, Shmerling $\mathrm{RH}$. The benefit of nonpharmacologic therapy to treat symptomatic osteoarthritis. Curr Rheumatol Rep. 2008;10: 510. DOI http://dx.doi.org/10.1007/s11926-008$\underline{0002-0}$

Lim JY, Tchai E, Jang SN. Effectiveness of aquatic exercise for obese patients with knee osteoarthritis: a randomized controlled trial. PMR. 2010;2(8): 723-31.

DOI http://dx.doi.org/10.1016/j.pmri.2010.04.004

Lund $H$, Weile $U$, Christensen R, Rostock $B$, Downey A, Bartels EM, Danneskiold-Samsøe B, Bliddal $\mathrm{H}$. A randomized controlled trial of aquatic and land-based exercise in patients with knee osteoarthritis. J Rehabil Med. 2008;40(2): 137-44. DOI http://dx.doi.org/10.2340/16501977-0134.

Pisters MF, Veenhof C, Schellevis FG, De Bakker $\mathrm{DH}$, Dekker J. Long-term effectiveness of exercise therapy in patients with osteoarthritis of the hip or knee: a randomized controlled trial comparing two different physical therapy interventions. Osteoarthritis Cartilage. 2010;18(8): 1019-26. DOI http://dx.doi.org/10.1016/j.joca.2010.05.008

Rannou F, Poiraudeau S. Non-pharmacological approaches for the treatment of osteoarthritis. Best Pract Res Clin Rheumatol. 2010;24(1): 93106.

http://dx.doi.org/10.1016/j.berh.2009.08.013

Reeuwijk KG, Rooij M, Van Dijk GM, Veenhof C, Steultjens MP, Dekker J. Osteoarthritis of the hip or knee: which coexisting disorders are disabling? Clin Rheumatol. 2010;29(7):739-47. DOI http://dx.doi.org/10.1007/s10067-010-1392-8

Silva LE, Valim V, Pessanha APC, Myamoto S, Jones A, Natour J. Hydrotherapy versus conventional land-based exercise for the management of patients with osteoarthritis of the knee: a randomized clinical trial. Phys Ther. 2008;88(1): 12-21.

van Dijk GM, Veenhof C, Schellevis F, Hulsmans H, Bakker JP, Arwert H, Dekker JH, Lankhorst GJ, Dekker J.Comorbidity, limitations in activities and pain in patients with osteoarthritis of the hip or knee. BMC Musculoskelet Disord. 2008;9: 95. DOI http://dx.doi.org/10.1186/1471-2474-9-95
Wang TJ, Belza B, Thompson F, Whitney JD, Bennett K. Effects of aquatic exercise on flexibility, strength and aerobic fitness in adults with osteoarthritis of hip or knee. J Adv Nurs. 2007;57(2): 141-52.

Wyatt FB, Milam S, Manske RC, Deere R. The effects of aquatic and tradicional exercise programs on persons with knee osteoarthritis. J Strength Cond Res. 2001;15(3): 337-40.

Yurtkuran M, Yurtkuran M, Alp A, Nasircilar A, Bingöl U, Altan L, Sarpdere G. Balneotherapy and tap water therapy in the treatment of knee osteoarthritis. Rheumatol Int. 2006;27(1): 19-27. DOI http://dx.doi.org/10.1007/s00296-006-0158-8 